\title{
TMS - Transcranial Magnetic Stimulation in medicine
}

\section{TMS - Stimolazione Magnetica Transcranica in medicina}

\section{TMS - Estimulación Magnética Transcraneal en medicina}

\author{
Vincenzo M. Mastronardi, ${ }^{1}$ Monica De Vincentis ${ }^{2}$ \\ ${ }^{1}$ Psichiatra, Psicoterapeuta, Ipnologo Clinico, Docente di Psichiatria Corso di laurea inf. Canale H Università di Roma \\ Sapienza; ${ }^{2}$ Laureanda in Investigazione, Criminalità e Sicurezza Internazionale, Italy
}

\begin{abstract}
TMS, that stands for Transcranial Magnetic Stimulation, is an innovative therapy based on electromagnetic stimulation of the nervous system. Although initially invented for neuropsychiatric pathologies, recent studies have proven it to be applicable to other areas of medicine as well, including oncology. It is now since 1971 that Vincenzo M. Mastronardi dedicates to research about the potential presence of electromagnetic messages in healthy organs (as all organs send electric and electromagnetic waves: skin, liver, kidneys, brain, prostate, etc.). Recording said messages and entering them with peculiar vehicular strategies in a more receptive organic state, like electromagnetic fields, clinic hypnosis, electrosleep, etc., it could be possible to contribute to the organ's recovery. Nowadays, evolved studies have taken their first steps from this assumption. The modern uses of electromagnetic waves employed in the clinical field in multiple universities and hospitals are going to be briefly resumed here.
\end{abstract}

\section{RIASSUNTO}

La TMS, ovvero Stimolazione Magnetica Transcranica, è una terapia innovativa basata sulla stimolazione elettromagnetica del sistema nervoso. Sebbene in origine fosse stata ideata per trattare patologie di ordine neuropsichiatrico, recenti studi ne hanno dimostrato la possibile applicazione in diverse aree della medicina, tra cui l'oncologia. È ormai dal 1971 che Vincenzo M. Mastronardi si dedica alla ricerca di eventuali messaggi elettromagnetici dell'organo sano (tutti gli organi inviano onde elettriche ed elettromagnetiche: pelle, fegato, reni, cervello, prostata, ecc.). Registrando tali messaggi ed immettendoli con peculiari strategie di veicolo in uno stato di maggiore recettività dell'organismo, quali per esempio campi elettromagnetici, elettrosonno, particolari strategie di ipnosi clinica, ecc., possiamo contribuire alla guarigione di quell'organo. Gli studi ormai evoluti partirono da questo assunto. Verranno ripresi in questa sede, sia pure in breve, gli attuali utilizzi delle onde elettromagnetiche impiegate ormai in ambito clinico in più Università e strutture ospedaliere.

\section{RESUMEN}

La TMS, o sea Estimulación Magnética Transcraneal, es una terapia innovadora basada en la estimulación electromagnética del sistema nervioso. Aunque en el comienzo fue ideada para tartar trastornos neuropsiquiátricos, recientes estudios han demostrado su aplicabilidad en otras áreas de la medicina también, incluso la oncología. Desde 1971, Vincenzo M. Mastronardi ha dedicado sus investigaciones científicas a la presencia potencial de mensajes electromagnéticos en órganos en salud (todos los órganos envían ondas eléctricas y electromagnéticas: piel, hígado, riñones, cerebro, próstata, etc.). Grabando estos mensajes e insiriéndolos comuna estrategia vehicular peculiar en un estado de órganos receptivos, como el campo electromagnético, le hipnosis clínica, el electro sueño etc., sería posible contribuir a la recuperación del órgano. Hoy en día, los estudios se han evolucionado a partir de estas asunciones. Los usos recientes de las ondas electromagnéticas empleadas en el campo clínico en varias universidades y hospitales serán resumidos en breve en este papel.

\section{Come funziona}

La stimolazione magnetica transcranica (TMS dall'inglese transcranial magnetic stimulation) si basa sulla stimolazione elettromagnetica del sistema nervoso. Permette lo studio dei circuiti e delle connessioni neuronali all'interno del cervello, attraverso una cosiddetta microlesione terapeutica che inibisce il funzionamento dell'area in esame. È in grado, in questo modo, di indurre e plasmare i fenomeni di riorganizzazione neuronale, facilitando o inibendo a seconda delle necessità terapeutiche, circuiti neuronali responsabili di una determinata area funzionale.

La strumentazione si avvale di una serie di coil (bobine di stimolazione) o elettrodi, posizionati sulla testa del paziente. Il coil produce energia elettrica che genera un campo magnetico a livello cerebrale per un breve periodo (cosiddetta lesione transitoria), inibendo le funzioni cognitive disfunzionali dell'area in questione. Il campo magnetico raggiunge quindi l'encefalo e le strutture cerebrali sottostanti.

Il coil si posiziona sul capo in corrispondenza della regione del cervello interessata. La variazione del campo magnetico $(2,5$ Tesla di intensità di $200 \mu$ s e $1 \mathrm{~ms}$ di durata) genera un flusso di corrente elettrica che interferisce sulla normale attività cerebrale determinando una depolarizzazione dei neuroni. Lo stimolo magnetico crea, dunque, una risposta sonora. La depolarizzazione neuronale può causare l'attivazione di gruppi di neuroni, indipendentemente dalla distanza dall'area stimolata. La stimolazione è praticata "per via transinaptica, partendo dalle fibre più sottili per poi raggiungere quelle più grosse dei primi strati della corteccia."1

F. Fiore (2017), "La stimolazione magnetica transcranica (TMS) - Introduzione alla Psicologia". https://www.stateofmind.it/2017/06/stimolazione-magnetica-transcranica/ 
Per stimolare strutture poste più in profondità, è possibile adoperare bobine di dimensione maggiore. Inoltre, la distanza della bobina dall'area interessata è inversamente proporzionale all'intensità del campo elettrico.

L'effetto può classificarsi come:

- “Attivazione (induzione/incremento dell'attività elettrica di un'area altrimenti inattiva o ipoattiva);

- Inibizione (riduzione dell'attività elettrica in corso in una determinata area)"2

\section{Storia}

Precedentemente alla TMS, la stimolazione elettrica era utilizzata, direttamente sui nervi e sui muscoli. Quest'ultima fu impiegata per la prima volta nel $\mathbf{1 7 9 0}$ da Galvani e Volta, per rendere eccitabili le membrane: tramite elettrodi, la corrente elettrica depolarizza le cellule e ne facilita la comunicazione. Oggi, è usata per misurare la velocità di conduzione dei potenziali di azione dei neuroni e per favorire la stimolazione dei muscoli compromessi, con parametri di $20 \mathrm{~mA}$ per $100 \mu \mathrm{sec}$, raggiungendo i 250 volt necessari per guidare la corrente elettrica attraverso la pelle.

Gli svantaggi della stimolazione elettrica possono riassumersi in questo modo:

- Fastidio dovuto al passaggio della corrente;

- Difficoltà nella stimolazione cerebrale profonda;

- Resistenza elettrica di alcune parti del cervello.

Una terapia alternativa è stata creata sfruttando campi magnetici a tempo variabile, basando gli studi sui principi dell'induzione elettromagnetica, scoperti da Michael Faraday nel 1831 e utilizzati per la stimolazione cerebrale già nel XX secolo.

Nonostante i primi tentativi non ebbero successo, nel $1976 \mathrm{fu}$ avviato un progetto nel Regno Unito, dall'Ospedale Royal Hallamshire, finalizzato alla stimolazione dei nervi periferici attraverso le correnti indotte da un campo magnetico, di breve durata tale da rilevare la risposta elettrofisiologica risultante. La TMS fu per la prima volta presentata ed utilizzata del 1985, in ambito diagnostico e terapeutico.

Il termine "magnetico" è, però, inesatto. Infatti, il meccanismo alla base della TMS è di tipo elettrico, sebbene contribuisca alla generazione di un campo magnetico nel paziente.

\section{Terapie}

La durata di una terapia di TMS è di pochi minuti, e comunque solitamente meno di un'ora. Durante la terapia, il paziente è sveglio e cosciente, e l'operatore ha il compito di rivolgergli alcune domande per controllarne le funzioni cognitive, tra cui emozioni e sensazioni fisiche, dopo una anamnesi mirata alla anamnesi,

2 S. Pallanti (2020), "Stimolazione Magnetica Transcranica ripetitiva (rTMS)". https://www.stefanopallanti.com/servizi/cosa-sono-le-terapiedi-neuromodulazione/rtms/

3 S. Pallanti (2020), "Stimolazione Magnetica Transcranica ripetitiva (rTMS)". https://www.stefanopallanti.com/servizi/cosa-sono-le-terapiedi-neuromodulazione/rtms

4 Redazione Aboutpharma Online (2019), "Tumori, il termomagnetismo come supporto alle cure oncologiche". https://www.aboutpharma. com/blog/2019/06/20/tumori-il-termomagnetismo-come-supporto-allecure-oncologiche/ assunzioni farmacologiche e variazioni nelle abitudini di vita quali per es. assunzione di alcool e/o sostanze, che possono interferire con le TMS.

Il paziente si presta, in media, ad 1 o 2 sedute giornaliere, per cicli di minimo 20 sedute (negli Stati Uniti il primo ciclo somministrato si aggira attorno alle 40 sedute), che avvengono sotto supervisione costante. Se il ciclo di sedute produce effetti benefici, di norma viene ripetuto.

\section{Applicazioni}

Attraverso sperimentazioni, la TMS si è rivelata efficace per: dolori di vario genere, disturbi del movimento, ictus, sclerosi laterale amiotrofica, sclerosi multipla, epilessia, depressione, disturbi d'ansia, disturbi ossessivo compulsivi, schizofrenia e dipendenze.

L'utilizzo di questa terapia ha ricevuto l'approvazione della Food and Drug Administration (FDA) circa il suo utilizzo come trattamento per l'emicrania. La TMS ripetuta (rTMS) invece, costituita dal ripetersi, appunto, di più cicli, è impiegata nel trattamento della depressione, nel caso in cui altri trattamenti e/o farmaci si rivelassero insufficienti. In ambito clinico, è utilizzata per il miglioramento della circolazione cerebrale e delle funzioni cognitive.

Recenti studi hanno comprovato come pazienti colpiti da patologie neurologiche sottoposti a rTMS per motivi diagnostici, e con disturbi dell'umore, trovassero giovamento in ambito depressivo. Ciò ha permesso pertanto l'implementazione di questa terapia in ambito neuropsichiatrico.

In particolare, ad oggi gli usi della TMS possono essere così riassunti:

In psichiatria:

- Depressione resistente ai farmaci

- Diabete

- Depressione post-partum

- Obesità

- Depressione associata con disturbi metabolici

- Depressione in cui i farmaci debbano essere evitati (insufficienza epatica, renale. etc.)

Autismo

- Psicosi

- Allucinazioni uditive

- Disturbo ossessivo-compulsivo

In neurologia:

- Riabilitazione cognitiva

- Riabilitazioni post stroke o trauma cranico

- Dolore, incluse le cefalee

- Parkinson

- Tinnitus (acufene)

Nei disturbi del peso e della nutrizione:

- Craving glucidica (fame di carboidrati)"3

Studi condotti dal Politecnico di Torino e l'Università di Torino ${ }^{4}$ sono stati dedicati all'applicazione della TMS per il trattamento di tumori, in abbinamento a terapie già esistenti. In particolare, si tratta di un'irradiazione delle cellule tumorali con onde elettromagnetiche a bassa frequenza, calcolata attraverso analisi termofisiche che combinano fisica e termodinamica e si basano sul principio fondamentale dell'induzione elettromagnetica o Legge di Faraday. Lo scopo è rallentare la proliferazione della cellula cancerogena, che in questo caso spenderebbe le proprie energie per "combattere" le onde o per meglio dire "per difendersi dalle onde" piuttosto che per crescere e diffondersi all'interno dell'organismo. 
Inoltre, la TMS si è dimostrata utile nel trattamento dell'ADHD (disturbo da deficit di attenzione e iperattività). Si tratta di un disturbo neurobiologico caratterizzato da iperattività, inattenzione e impulsività. Attraverso studi focalizzati sulle neuroimaging sono state osservate alterazioni nel circuito fronto-striatale, soprattutto nell'emisfero destro (Castellanos e Acosta, 2002; Arnsten, 2006).

Il trattamento farmacologico per l'ADHD può essere problematico per alcuni individui e non scevro da effetti collaterali. Per questa ragione la Stimolazione Transcranica Magnetica si presenta come una soluzione valida in molti casi, essendo uno strumento poco invasivo ed indolore (Acosta et al., 2002; Hallett, 2001; Siebner e Rothwell, 2003; Strafella et al, 2001). La rTMS si è dimostrata efficace per il trattamento di disturbi inerenti all'anomala attivazione di alcune aree cerebrali attraverso la stimolazione del circuito disfunzionale fronto-striato-cerebellare, unitamente al training cognitivo (Demirtas-Tatlidede et al, 2013). Il trattamento rTMS per l'ADHD consiste in $\mathbf{1 5}$ sessioni a $1 \mathrm{~Hz}, 1200 \mathrm{stim} / \mathrm{die}$.

\section{Apprendimento durante il sonno}

Le nostre ricerche sull'apprendimento durante il sonno sono state da noi (Mastronardi) eseguite presso la Clinica Neurologica dell'Università di Bari al ben preciso scopo di verificare la maggiore recettività psicofisica del nostro organismo nei momenti di slivellamento dello stato di coscienza e quindi facendo fruttare tutte le precedenti ricerche già da noi effettuate con Franco Granone durante il sonno ipnotico presso l'Ospedale S. Andrea di Vercelli nel trattamento di varie patologie e di diversi comportamenti disfunzionali quali ad es alcoolismo e tossicodipendenza. Per sonno parziale può intendersi quello stato in cui l'inibizione della coscienza non è totale ma relativa, conservando il soggetto un certo rapporto col mondo esterno, analogamente a quanto avviene per il viaggiatore, o per il telefonista e per la balia, che pur riuscendo a dormire in mezzo al frastuono si svegliano al più piccolo rumore riguardante il proprio compito. Analoghe affermazioni ci vengono dal fenomeno dell'autista che guida dormendo o dell'ormai noto "dormire durante la marcia" dei soldati stanchi. A questo tipo di sonno, quindi, sarebbe da paragonarsi il sonno ipnotico o quello del T.A. che sarebbe perciò "sonno parziale artificiale indotto, con ottundimento dei poteri di critica e limitazione del campo della coscienza".

Diversi autori hanno dimostrato che durante la fase di sonno REM può verificarsi quello che viene chiamato apprendimento dipendente dallo stato (state dependent learning), un fenomeno che Overton (1972) ha riscontrato nello stato di ebrezza portato da diversi medicamenti. Interessanti anche le sperimentazioni di Evans e collaboratori (1966) che hanno dimostrato come durante la fase REM possono essere impartite istruzioni anche complesse. Secondo una teoria onirica (Bergson) indipendentemente dai sogni con funzione di salvaguardia del sonno, da quelli con funzione di realizzazione dei desideri e quelli contenenti materiali infantili, i sogni spesso vengono a strutturarsi per effetto di stimoli esterni al soggetto che dorme. La possibilità che stimoli esterni, presentati durante il sonno rem, siano incorporati nei sogni è stata riferita per il suono, il lampo di luce, soprattutto il getto d'acqua sulla pelle e per i nomi di persone.

Jessen riferendo dei sogni che possono essere ricondotti ad eccitazioni sensoriali oggettive più o meno accidentali quot iscrive: "ogni rumore percepito indistintamente desta corrispondenti immagini oniriche. Il fragore di un tuono ci collocherà nel mezzo di una battaglia; il canto del gallo può trasformarsi nel grido di ter- rore di un uomo; il cigolio di una porta può produrre un sogno di ladri. Se la nostra coperta cade durante la notte, potremmo sognare di camminare nudi o di cadere nell'acqua. Se siamo stesi di traverso sul letto e spingiamo i nostri piedi al di là del bordo, possiamo sognare che siamo sull'orlo di uno spaventoso precipizio o che stiamo cadendo da un dirupo. Se la nostra testa per caso va a finire sotto il cuscino, sogniamo di essere sotto una gigantesca roccia che ci sovrasta e sta per seppellirci sotto il suo peso. L'accumularsi del seme porta sogni voluttuosi, dolori locali portano l'idea di maltrattamenti, attacchi o ferite che ci vengono fatti in quel momento...".

Meier sognò una volta di essere sopraffatto da alcune persone che lo stesero a terra sulla schiena e conficcarono nel terreno un palo tra il suo alluce e l'indice. Mentre immaginava tutto questo nel sogno, si svegliò e scoprì che un filo di paglia si era inserito fra le dita del suo piede. Un'altra volta secondo Hennings Meier chiuse la sua camicia troppo stretta intorno al collo e allora sognò che lo stavano impiccando. Hoffbauer sognò quando era giovane di cadere un alto muro, poi quando si svegliò si accorse che la rete del letto aveva ceduto e che era veramente caduto a terra.

Koella, che parla di MB (memoria breve) e ML (memoria lunga) a proposito del processo di consolidamento nella memoria di materiale acquisito durante il sonno, già scriveva che l'informazione viene recepita attraverso i nostri organi sensoriali ed avviata al Sistema Nervoso Centrale e che la ML è di natura materiale e probabilmente si basa sulla sintesi di materiale proteico complesso ed altamente specifico, e aggiunge: "la diminuzione dell'attività delle cellule nervose (p. es. nel coma e nella narcosi) non influenza questo materiale. Il blocco del processo di consolidamento (sperimentalmente per es. mediante disattivazione dell'enzima necessario per la fabbricazione delle proteine) fa perdere il materiale depositato nella MB". In tema di "Behaviour Therapy" durante il sonno rem in alcolisti cronici, riferiamo le prime sperimentazioni condotte da Puca, Mastronardi, Minervini, Passarella, Vitolo, presso la clinica neurologica dell'università di Bari.

La casistica era inizialmente formata da quattro alcolisti cronici di età compresa tra 35 e 39 anni, tre uomini ed una donna. La terapia comportamentale, effettuata mediante riproduzione di audiocassette, consisteva in avversione (Adversion therapy) evocata con l'immaginazione, secondo tecnica detta di sensibilizzazione nascosta. (Cautela). L'avversione evocata era talvolta rafforzata dalla somministrazione di stimoli olfattivi sgradevoli (ammoniaca) e, alternativamente alle immagini avversative, venivano evocate immagini di benessere e sicurezza. Le audiocassette erano personalizzate per i casi 1 e 2, mentre i casi 3 e 4 hanno iniziato il trattamento con audiocassette non personalizzate. Dopo due notti di ambientamento, si registrava poligraficamente il sonno. Si riproduceva l'audiocassetta quando il paziente era, da almeno due minuti, inequivocabilmente in sonno rem, interrompendo la riproduzione quando si verificava un cambiamento di fase o un risveglio. Le audiocassette erano riprodotte in successione numerica. Ai risvegli venivano annotati i ricordi dei sogni. I pazienti erano stati volutamente informati soltanto del fatto che, nel corso della notte, si sarebbe cercato di rafforzare la loro volontà ad astenersi dall'alcol. In effetti, un caso, risvegliatosi durante la riproduzione di una cassetta, ed un altro, che dopo l'ambientamento, ha voluto conoscere le modalità della terapia, sono stati necessariamente messi al corrente di tutte le particolarità della terapia comportamentale. Per escludere che i risultati fossero dovuti solo ad effetto suggestivo, per 7 notti, in altri due alcolisti, in trattamento psicoterapeutico, sono state riprodotte, in sonno rem, audiocassette che non suscitavano immagini avversative nei riguardi dell'alcol.

L' avversione all'alcol si è verificata in tutti i 4 casi. Nel caso 1 dopo tre notti di trattamento, nel caso 2 dopo due notti, nel caso 3 dopo cinque notti (nelle prime tre notti audiocassette non perso- 
nalizzate), nel caso 4 dopo sei notti (nelle prime tre notti audiocassette non personalizzate), ma il risultato positivo è apparso in relazione alla personalizzazione delle immagini suscitate dalla terapia avversativa verbale. Tra queste, infatti, sembrava venissero recepite efficacemente soprattutto quelle pregnanti di significati per il paziente dato che durante la loro riproduzione si verificavano modifiche comportamentali e poligrafiche, come sobbalzi, sussulti, cambiamenti di posizione, aumento dei REMs e del tono muscolare e che tracce di tali immagini si rinvenivano talora nei racconti dei sogni. Sono rimasti astemi dopo la fine del trattamento: il caso 1 per quattro mesi, il caso 2 per tre mesi, il caso 3 per un mese, il caso 4 per 20 giorni. A distanza di 7 mesi dalla fine del trattamento, il caso 1 beve saltuariamente quantità minime di alcol, il caso 2 dopo sei mesi beve occasionalmente; a circa 5 mesi di distanza il caso 3 è tornato alle abitudini precedenti la terapia ed il caso 4 beve saltuariamente quantità ridotte.

I risultati di questa ricerca confermano la possibilità di apprendimento inconscio, durante il sonno REM (Cooper e Hoskovec) e indicano, che, durante tale tipo di sonno, è possibile effettuare con successo la terapia comportamentale dell'alcolismo. L'avversione all'alcol si è infatti osservata in tutti i casi, due dei quali non erano al corrente (a livello cosciente) della metodica adoperata. Dalle nostre osservazioni emerge però la necessità che le immagini suscitate con la terapia avversativa verbale per essere efficacemente recepite durante il sonno REM, debbano essere sufficientemente personalizzate. Infatti, la reazione avversativa all'alcol non compariva quando le immagini suggerite dalle audiocassette non erano individualizzate, tanto che per ottenere il risultato positivo è stato necessario nei casi 3 e 4 modificare la tecnica in tal senso. Una maggiore efficacia sull'alcolismo di una terapia comportamentale individualizzata rispetto ad una non individualizzata è stata d'altronde segnalata anche in veglia da Sobell e Sobell. Pertanto, la terapia comportamentale in corso di sonno rem può essere effettuata in un centro contro l'alcolismo; necessita, però, per essere efficace di individualizzazione della metodica e, al fine di un risultato più stabile, di integrazione con un valido e continuo rapporto psicoterapico allo stato di veglia, come in tutti i trattamenti avversativi (Deveny e Sereny). Rispetto ai risultati ottenuti con analoga metodica durante ipnosi, quelli osservati in corso di sonno REM compaiono più precocemente, dato che durante ipnosi sono necessarie almeno 5 o 6 sedute per ottenere l'avversione. In confronto ai trattamenti effettuati in ipnosi restano da valutare i problemi finanziari anche se, nell'eventuale sviluppo di utilizzazione di tali tecniche, il costo di una notte di registrazione, per personale, apparecchiatura, consumo di carta ed altro, potrebbe venire superato da un eventuale riconoscimento automatico del sonno REM con accensione automatica del registratore che riproduce l'audiocassetta.

In seguito, la casistica fu estesa a 6 alcolisti, 5 uomini e una donna con età tra 35 e 45 anni, e riferiamo qui di seguito lo studio particolareggiato dei sogni condotto dagli stessi Puca-PassarellaMastronardi-Vitolo-Minervini. Nei primi 4 casi è stata condotta per 6-8 notti una terapia comportamentale dell'alcolismo, mediante riproduzione di audiocassette, consistente in avversione evocata con l'immaginazione secondo tecnica di sensibilizzazione nascosta (cautela). L' avversione era talvolta rafforzata da stimoli olfattivi sgradevoli (ammoniaca) ed alternativamente ad essa venivano evocate anche immagini di benessere e sicurezza. I casi 5 e 6 sono stati adoperati come controlli dei primi 4 ai fini della terapia comportamentale; le audiocassette riprodotte durante il loro sonno per sei notti non evocavano, pertanto, immagini capaci di indurre avversione all'alcol. Esaminando la registrazione poligrafica del sonno, si riproducevano in successione numerata le audiocassette, una per ciascun episodio di sonno rem, quando il soggetto era da almeno due minuti in inequivocabile sonno rem. Al mattino e ad ogni risveglio notturno spontaneo veniva richiesto se, quante volte e cosa avessero sognato ed il ricordo dei sogni veniva annotato. Per facilitare il rilievo di eventuali correlazioni tra il racconto dei sogni ed il contenuto delle audiocassette, gli elementi essenziali di queste sono stati riportati per ciascun caso nei risultati, così come (sono stati riferiti nei risultati) cenni anamnestici, quando significativi, per l'interpretazione di elementi onirici.

\section{Caso n. 1}

Le immagini salienti delle 4 audiocassette riprodotte nel corso di otto notti sono: nella prima, donna trasandata, nauseante, abbrutita dall'alcol, prelevata da infermieri che, in ambulanza, la trasportano in Ospedale Psichiatrico; nella seconda, due alberi, uno solido e vedreggiante, l'altro esile e secco con rami penduli; nella terza, il paziente, adagiato sul divano e pervaso da un profondo benessere, diventa leggero e si solleva per ritrovarsi in un ameno giardino con fiori ed uccellini che cinguettano in riva ad un ruscello; nella quarta, l'albero della vita, robusto e vigoroso, slanciato verso l'alto, dalle cui liane si attinge la linfa vitale. Nei racconti dei sogni dalla prima, seconda, e quinta notte vi è una figura femminile, i cui tratti, pur mutevoli nei vari racconti, ben s'identificano con l'immagine della donna ubriaca (cassetta 1 ); nella quinta notte la donna acquista il volto a lui (paziente) noto di colei che, deludendolo, gli comportò l'abuso di alcol. Nel racconto della settima notte riferisce di essersi sentito sollevare dal piano del letto, provando una sensazione di piacere (cassetta 3). Nella ottava notte il paziente si trova in un giardino, al centro del quale vi è una "strana macchina parlante" che egli chiama "albero della vita" (cassetta 4). Nel corso dell'ottava notte riferisce anche di aver sognato, prima di svegliarsi nauseato, di accingersi a bere birra e nella prima notte di venire sottoposto a registrazione poligrafica notturna. Notevoli variazioni comportamentali (sobbalzi, sussulti, cambi di posizione, modificazioni del tono muscolare, accentuazione dei movimenti oculari) sono state osservate durante la riproduzione di alcune frasi delle cassette 1 e 6 (Fig. 1).

\section{Caso n. 2}

Le immagini salienti delle 4 audiocassette riprodotte nel corso di sei notti sono: nella prima, il cimitero al crepuscolo dove, presso la sua tomba, incontra il padre che le parla, infondendole sicurezza e fiducia; nella seconda, una festa da ballo, dove le viene impedito di ballare dalla madre, con il pretesto di non essere vestita ordinatamente, nella terza e nella quarta, rispettivamente la prima e la terza del caso 1 . Si evidenziano tracce dei messaggi contenuti nella cassetta 1 , nel racconto dei sogni della prima notte (un medico anziano, buono e generoso, le infonde fiducia e sicurezza) della terza notte (rapporti sessuali con un signore anziano, lontano parente che le ricorda il padre) e della quinta notte (nel cimitero, dove si è recata per visitare la tomba del padre, nota che i cancelli ed i viali sono diversi e non trova più la tomba del padre; dopo un risveglio angoscioso il sonno riprende nello stesso cimitero, ove, ai piedi di una scalinata, ritrova la tomba paterna ricoperta di fiori ed il padre in sua attesa elegantemente vestito, in compagnia di distinti signori; egli la tranquillizza e la esorta a cambiare vita e ad affrontare con maggiore coraggio le difficoltà). Tracce dei messaggi della cassetta 2 si rinvengono nel racconto dei sogni della quarta notte (in una scuola da ballo è ostacolata dall'insegnante, che non le vuole impartire la lezione, pur pretendendone il pagamento) e della quinta notte (ad una festa vorrebbe ballare, ma ne è impedita da qualcuno che non sa riconoscere). Una figura di donna sgradevole (cassetta 3 ), spesso identificata con la madre, viene anche riferita nei sogni della prima, seconda, quinta notte. La registrazione poligrafica nottturna è sognata nella seconda notte. Chiare modificazioni comportamentali sono state determinate dalla riproduzione 
di un nastro contenente alcune frasi di particolare risonanza emotiva per la paziente.

\section{Caso n. 3}

Le immagini evocate dalle audiocassette durante 7 notti sono per la prima, seconda, terza, simili rispettivamente alla seconda, quarta, prima del caso n.1; per la quarta scena di mare, di pesca, di vacanza familiare. Riferisce di non aver mai sognato, tranne che la seconda notte, quando racconta di essere stato "spettatore in un sogno" della propria esperienza di registrazione notturna. Discrete modificazioni comportamentali hanno talora fatto seguito alla riproduzione di alcuni brani delle audiocassette (Fig. 2).

\section{Caso n. 4}

Le immagini evocate dalle cassette durante 7 notti sono per la prima, seconda, terza corrispondenti alla seconda, quarta, prima del caso n. 1; per la quarta: un giardino rigoglioso, l'arcobaleno dopo la pioggia, la bellezza della natura che infonde voglia di vivere. Il paziente non ha mai riferito alcun sogno. Non si sono mai osservate modificazioni comportamentali degne di nota durante la riproduzione dei nastri.

\section{Caso n. 5}

Le immagini contenute nelle cassette riprodotte durante 6

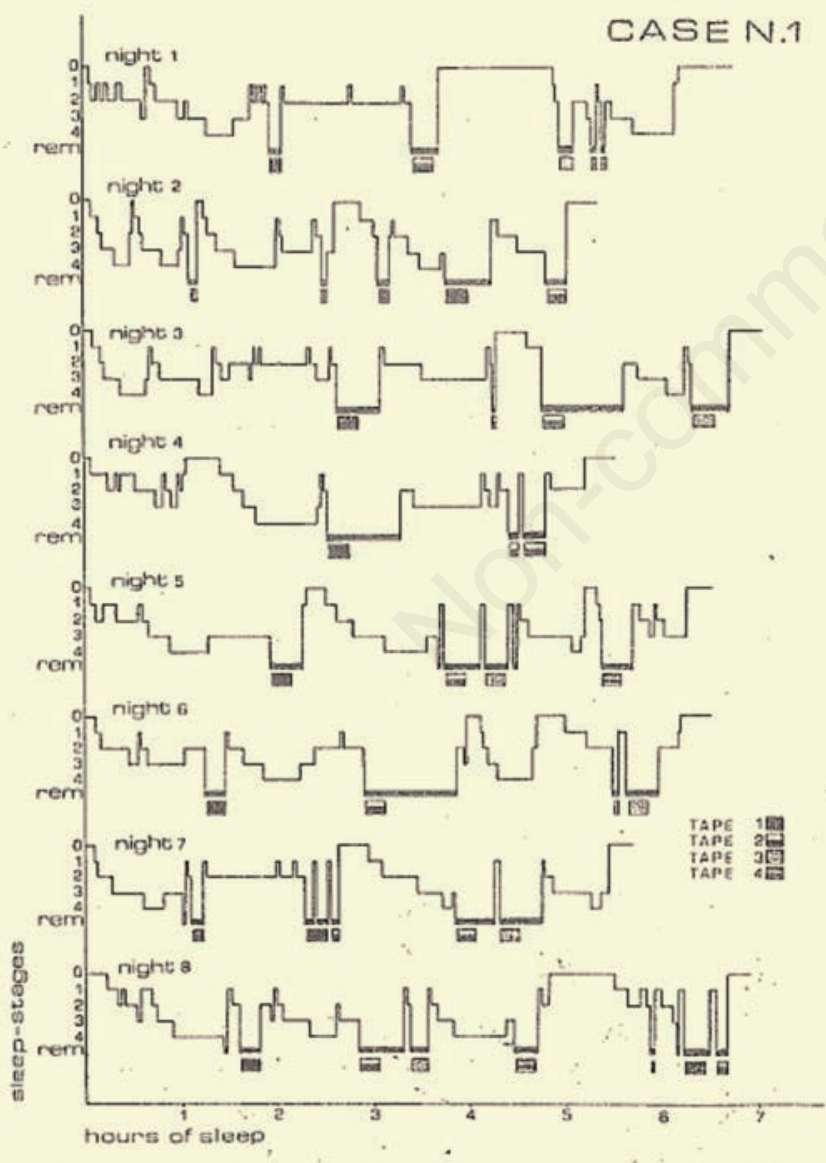

FiG. 1. - Ipnogrammi delie 8 notti nelle quali è stato attuato il trattamento del caso n. 1. Tape 1, 2, 3, 4 rappresentano le audiocassette mediante le quali veniva effettuata ta terapia avversiva evocata con timmaginazione. Dopo la
la presentato repulsione alla vista degli alcoolici.

Fig. 1. notti; per la prima, un'omicidio in un bosco: un uomo avvolto in un mantello nero sbuca da dietro un albero ed assale, pugnalandolo, un ignaro viandante, poi fugge; per la seconda, una ragazza nuda che si bagna in una fontana e con fare provocante invita a seguirla, ma vi sono numerose persone che si accalcano per guardare; per la terza, l'atterraggio su un lago, di sera, di un disco volante dal quale scendono alcuni extreterrestri; per la quarta, l'invito a correre su un prato verde ai margini di un bosco, sudando e riprendendo vigore fisico. Nel racconto dei sogni, un rapporto con il contenuto della prima ccassetta si rinviene la prima notte (in una foresta un puma gigantesco, "grande come King Kong" si rizza all'improvviso sulle zampe posteriori ed uccide all'istante un puma più piccolo); con il contenuto della seconda cassetta la terza notte (quando sogna di trovarsi su una spiaggia, dove una splendida donna nuda si butta in acqua ed egli tenta di avvicinarsi, ma la scena cambia e si trova a cena con la moglie) e la quarta notte (in cui sogna di assistere alla TV ad uno spettacolo di spogliarello, che viene interrotto dall'arrivo della moglie). Sono state notate discrete modificazioni comportamentali in particolar modo durante la riproduzione dell'audiocassetta a contenuto erotico.

\section{Caso n. 6}

Le immagini evocate dalle cassette riprodotte durante 6 notti sono: nella prima, rapina in banca, con l'irruzione di uomini con il

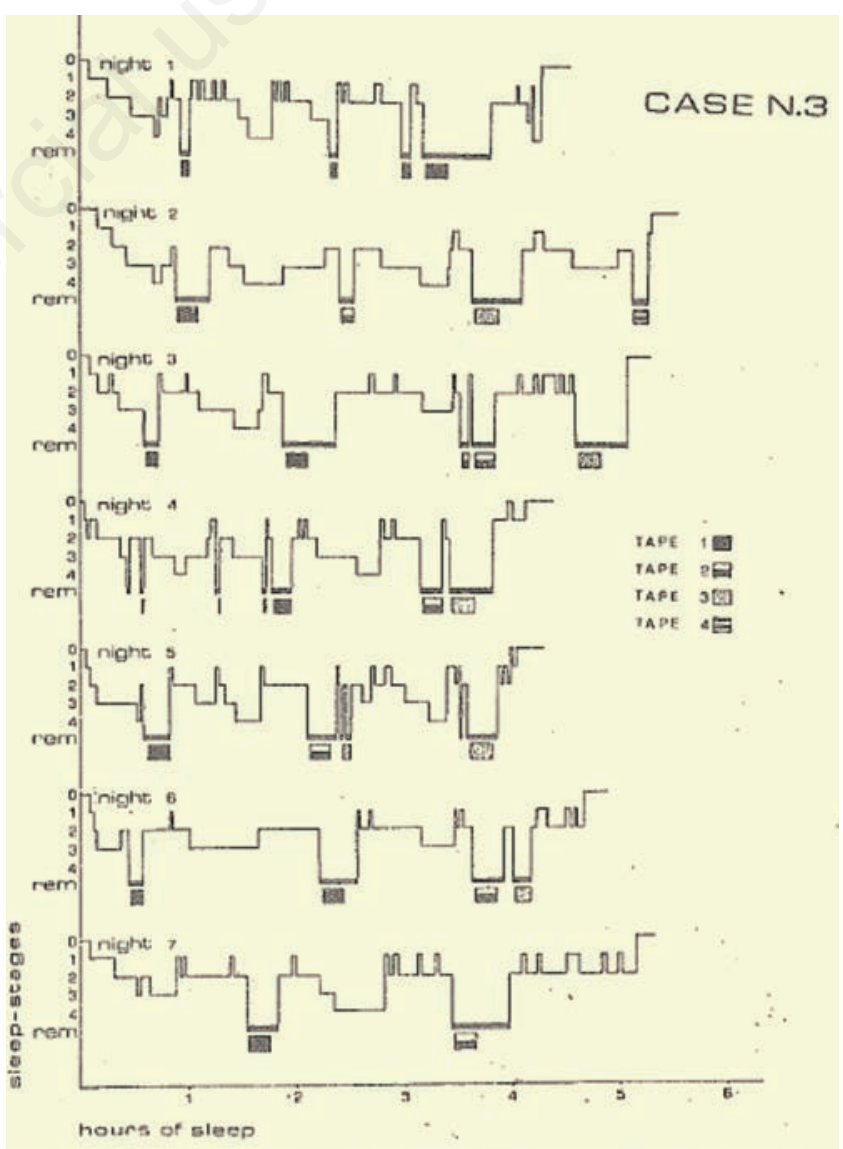

Fic. 2. - Ipnogrammi delle 7 notti in çui si è svolto il trattamerito del caso n. 3. Tape i, 2, 3, 4 rappresentano le audiocassette mediante le quali veniva effettuata la terapia avversiva evocata con $\mathrm{la}$ immaginazione. Nelle prime 3 notti sono state utilizzate audiocossette non e personalizzate s, come anche nel caso n. 4 , senzi alcur risultato. Dopo altre 2 notti-con audiocassette * personalizzate = si è ottenuta l'avversione afl'alcool.

Fig. 2. 
volto coperto, il terrore degli astanti, la consegna di tutti i valori da parte dei cassieri, la fuga con il bottino; nella seconda, terza, quarta come nel caso n. 5. Nel racconto dei sogni della seconda notte si rinvengono rapporti con il contenuto della prima cassetta (la banda di ladri, di cui egli fa parte, divide il bottino, frutto della rapina in un bosco), con la seconda cassetta la terza notte (in riva ad un lago ode voci femminili, poi scorge due ragazze che si bagnano nude ed allegre; vorrebbe avvicinarsi, ma avverte un senso di peso allo stomaco), con la quarta cassetta, riprodotta due volte prima del risveglio, la prima notte (in un bosco vicino ad un fiume un cavallo gli corre incontro ed egli per evitarlo si mette a correre a sua volta, sudando ed ansimando). Chiare modifiche comportamentali sono state osservate in seguito alle riproduzioni della seconda cassetta; poco dopo la fine di questa, il soggetto si è svegliato, riferendo di avere ancora "il sangue caldo".

\section{Considerazioni conclusive}

Da quanto evidenziato nei risultati è apparso chiaro che le immagini visive evocate in sonno REM possono, talora, influenzare il racconto dei sogni. Per esempio, nel caso n. 1 sono stati rinvenuti elementi contenuti nelle audiocassette: una figura di donna ripugnante, l'esperienza di sollevamento dal piano del letto, l'albero della vita. Nel racconto dei sogni del caso n. 2 la figura paterna, la tomba ed il cimitero, una donna sgradevole, poi identificata con la madre, le feste da ballo. Nel caso n. 5 l'uccisione improvvisa, la bagnante nuda, lo spogliarello. Nel caso n. 6 la rapina, la bagnante nuda, la corsa. Questi ed altri elementi meno chiaramente connessi alle immagini visive evocate, sono apparsi inseriti, variamente strutturati, nell'attività onirica già in corso. Le immagini visive esterne incorporate nei sogni, così come in un'altra esperienza (Berger), non sono state riconosciute con caratteri di estraneità. Discutibili sono, invece, apparsi gli effetti sui sogni dello stimolo olfattivo sgradevole (ammoniaca); infatti, nessuno dei soggetti ha riferito di avere avvertito odori sgradevoli, anche se talora è stato sognato un senso di nausea. Il sogno della propria esperienza di registrazione poligrafica è osservazione comune nei laboratori del sonno (Dement e Wolpert). La scarsità dei resoconti nei casi 3 e 4 può essere messa in relazione, oltre che con la minore collaborazione offerta da questi soggetti (Schombar), anche con il non aver presentato risvegli nel corso della notte, come appunto segnalato negli scarsi sognatori (Dement e Wolpert; Aserinski e Kleitman; Shevrin e Luborski; Whitman;Hartman). Infine, così come i risultati favorevoli della terapia comportamentale attraverso le immagini visive evocate, ottenuti in alcuni di questi soggetti (Puca et al.), anche il riscontro nei loro sogni di elementi contenuti nelle audiocassette sembra indicare chiaramente la possibilità di apprendimento inconscio durante il sonno REM.

Tutta la metodica utilizzata in questa sperimentazione fa fulcro

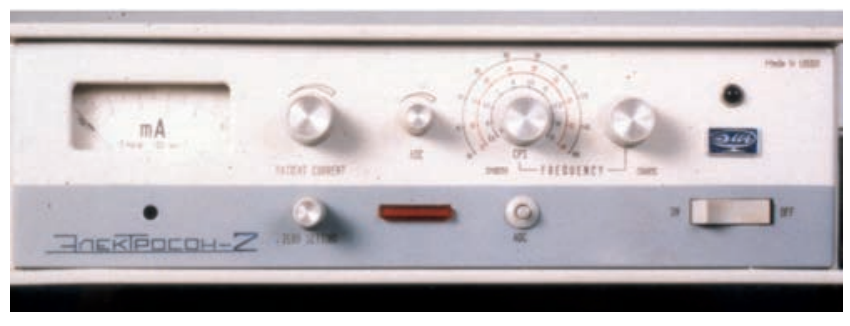

Fig. 3. sulle tecniche di condizionamento e decondizionamento utilizzata da Granone, riportate nel suo "Trattato di Ipnosi" e che ho appreso in qualità di Collaboratore durante i mesi di mia permanenza presso il Centro di Ipnosi Clinica-sperimentale Divisione Neurologica dell'Ospedale Generale "S. Andrea" di Vercelli nel 1975 dalla viva voce e dall'esperienza pluridecennale del Prof. Franco Granone stesso alla cui statura scientifica va tutta la mia indelebile gratitudine e la mia stima.

Sempre alla ricerca di metodiche che consentono l'eventuale esclusione della parte razionale e cosciente del nostro Io, e quindi l'accesso facilitato alla sua posizione inconscia, ho effettuato con la collaborazione del Dott. Santo Cerfeda alcune sperimentazioni direttamente dulla mia persona con l'intento di indurre sonno elettrico quindi artificialmente indotto mediante l'apparecchio "electric sleep" acquistato presso una ditta di articoli sanitari di Mosca (URSS) dall'Istituto di Criminologia e Psichiatria Forense dell'Università di Bari e ceduta quindi all'annessa Sezione di Ipnologia Clinica da me diretta (Fig. 3). Successivamente le sperimentazioni stesse sono continuate con diversi pazienti alcolisti e tossicomani con l'aiuto prestatomi dal tecnico, del suddetto Istituto, Mario Merlanti che ringrazio per la collaborazione.

Secondo la teoria di patologia cortico-viscerale di K. Bykov e secondo gli studi effettuati da Anokhin ed altri autori sovietici e non, e gli studi riguardanti la formazione reticolare (basati sulla teoria di Pavlov) qualsiasi malattia dovrebbe essere considerata non come una condizione patologica locale, ma come una condizione patologica dell'organismo nella sua interezza, con inevitabile disturbo delle funzioni del sistema nervoso centrale.

La corrente ad impulsi potrebbe essere considerata quale irritante, che simultaneamente produce effetti di controllo stimolante e protettivo sul sistema nervoso centrale e periferico. In tal caso l'effetto terapeutico si raggiunge attraverso la normalizzazione delle correlazioni fra corteccia e sezioni inferiori del cervello disturbate da processi patologici. Questo, alternativamente, varia la composizione fisico-chimica delle cellule nervose e migliora le funzioni trofiche dell'organismo. L'azione viene espletata grazie all'intervento di corrente ed impulsi, con frequenze da 3,5 a 11 Hertz, durata degli impulsi (fissa) a 0,5 metri al secondo, corrente alternata fino a 2,5 mA con una componente galvanica addizionale continua fino a $0,6 \mathrm{~mA}$ (Fig. 4).

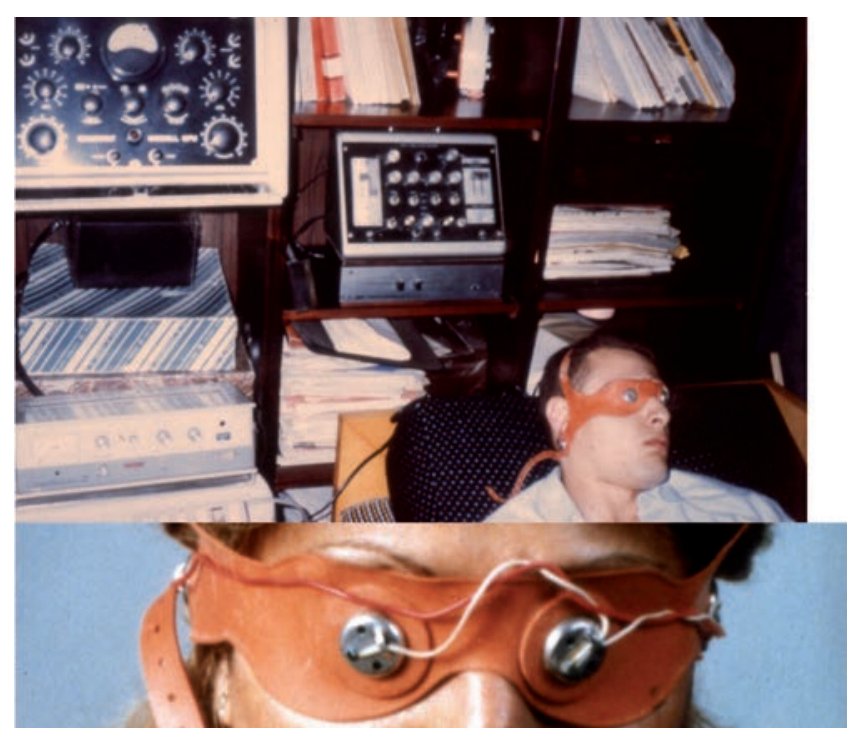

Fig. 4. 
Il dosaggio ottimale dell'intensità media di corrente sufficiente per provocare un sonno profondo è costituito dall'uso della forma combinata di corrente alternata da 0,8 a $1,5 \mathrm{~mA}$, e di corrente continua pari al $20-30 \%$ dell'alternata $(0,2-0,45 \mathrm{~mA})$, frequenza $100 \mathrm{~Hz}$, durata dell'impulso $0,5 \mathrm{~m} / \mathrm{sec}$ per 1 ora e 30 '. La cuffia permette agli impulsi elettrici di attraversare la zona del cervello interessata al sonno, grazie agli elettrodi situati sugli occhi e sull'area mastoidea.

Il sonno elettrico ha caratteristiche fisiologiche ben definite che lo distinguono dal sonno farmacologico e dalla narcosi elettrica. Se la tecnica è corretta, il sonno elettrico non genera sensazioni spiacevoli. Sotto controllo medico con frequenze da 60 a $135 \mathrm{~Hz}$ il paziente percepisce delicate vibrazioni o una leggera sensazione di senapismo nelle zone di applicazione degli elettrodi (area orbitale e mastoidea); dopo 5/15 minuti il paziente comincia a sentire pesantezza alle palpebre, il suo pensiero diventa confuso, appare un tenue languore ed il paziente si assopisce; nella maggior parte dei casi questo stato si trasforma in sonno profondo. Indipendentemente dall'azione diretta della corrente sul sistema nervoso e conseguente azione terapeutica della stessa in caso di insonnia, nevrosi, depressione, alcolismo, ecc., le sperimentazioni da me effettuate si riproponevano di osservare se durante le sedute di "Elettrosonno" analogamente a quanto già esaminato per il sonno REM fosse possibile la somministrazione di terapeutici condizionamenti ipnotici. Le ricerche hanno fin'ora convalidato che:

- Il sonno provocato dal trattamento con Electric sleep non interferisce con il sonno notturno; al contrario serve a prolungare quest'ultimo, a normalizzarlo e renderlo più profondo;

- Lo stato del paziente dopo il trattamento del sonno elettrico è caratterizzato nella maggior parte dei casi da un rimarchevole miglioramento dello stato generale e del tono dell'umore, nonché dalla abolizione dell'ansia. I pazienti riferiscono di sentirsi più "attivi ed ottimisti". In principio, questi cambiamenti nella sfera neuropsichica del paziente continuano per un periodo di tempo relativamente breve, ma successivamente, dopo un certo numero di sedute con il sonno elettrico, tendono a stabilizzarsi;

- Lo stato ipnoidale elettricamente indotto, anche se tende in maniera oscillante a trasformarsi in sonno profondo, è idoneo a consentire la somministrazione di messaggi eteroipnotici con le relative tecniche terapeutiche a nostra disposizione;

Le ricerche sono continuate poi in diverse direzioni terapeutiche con le stesse modalità cliniche e migliori perfezionamenti tecnici e tecnologici. Rimandiamo alle successive pubblicazioni le naturali, fisiologiche evoluzioni delle stesse in corso di stimolazione elettromagnetica ancor più ad ampio spettro terapeutico. La polarizzazione sui messaggi d'organo, attualmente hanno la nostra priorità attentiva di ricerca.

\section{Bibliografia}

Aserinski E, Kleitman V, (1953). "Regularly occurring periods of eye motility concomitant phenomena during sleep", in Science $118,273-274$

Bergandia I, Luciab U, Grisoliab G, Granatac R, Gesmundoc I, Ponzettoc A, et al. (2019). The extremely low frequency electromagnetic stimulation selective for cancer cells elicits growth arrest through a metabolic shift. BBA - Molecular Cell Research

Berger R, (1963). Experimental modification of dream content by meaningful verbal stimuli. Brit. J. Psychiat 109;722-740.

Bergson H, (1919). L'energie spirituelle. Alcan, Parigi.

Bergson H, (1958). Saggi sui dati immediate della coscienza. Signorelli, Milano.
Bergson H, (1958). The world of dreams. Phylosophical library Inc., New York.

Bergson H, (1963). Saggi sui dati immediate della coscienza. Paravia, Torino.

Bykov KM, (1958). La corteccia cerebrale e gli organi interni. Feltrinelli, Milano.

Cautela JR, (1967). Covert sensitization. Psychol Rep 20:459-68.

Cooper LM, Hoskovec J, (1972). "ypnotic suggestions for learning during stage I REM sleep. Am J Clin Hypnosis 15:102.

Dement W, Wolpert EA, (1958). The relation of the eye movements, body motility and external stimuli to dream content. J Exp Psychol 55:543-53.

Devenyi P, Sereny G, (1970). Aversion treatment with electroconfitioning for alcoolis. Brit J Addict 65;289.

Di Lazzaro V, (2013). Brain Stimulation: Chapter 30. Biological effects of non-invasive brain stimulation. In: Handbook of Clinical Neurology. p. 116

Electrosleep Therapy (NCD 30.4) UnitedHealthcare ${ }^{\circledR}$ Medicare Advantage PolicyGuideline Guideline Number: MPG092.06 Approval Date: August 12, 2020. https://www.uhcprovider. com/content/dam/provider/docs/public/policies/medadv-guidelines/e/electrosleep-therapy.pdf

Evans FJ, Gustafson LA, O'Connell DN, Orne MT, Shor RE, (1966). Response during sleep with intervening waking amnesia. Science 152:666-7.

Fiore F, (2017). La stimolazione magnetica transcranica (TMS) Introduzione alla Psicologia. https://www.stateofmind.it/ 2017/06/stimolazione-magnetica-transcranica/

Granone F, (1961). L'ipnotismo: considerazioni neuro-fisiologiche, psicologiche, terapeutiche, tratte da esperienze personali. Annali Freniat 74.

Granone F, (1961). Lo stato di coscienza in ipnosi. Atti Riunioni Med-Chir Intern Torino, 1961, Annali Freniat 74.

Granone F, (1962). L'ipnotismo come fenomeno biologico, mezzo d'indagine, strumento teraputico. Boringhieri, Torino.

Granone F, (1963). L'EGG negli stati ipnotici. Riv Neurol 33: 411.

Granone F, (1965). Modalità di tecnica ipnotica per il divezzamento degli etilisti. Annali Freniat 78:479.

Granone F, (1966). Ipnotismo nella cura delle psiconevrosi. Rass Ipnosi Med Psicosom 57:3633.

Granone F, (1967). Hypnotism in treatment of chronic alcoholism. Annali Freniat 80:145.

Granone F, (1967). Ipnotismo e medicina psicosomatica. Gazz Sanit 38.

Granone F, (1967). L'importanza dei rapporti psicosomatici in terapia ipnotica. Rass Ipnosi Med Psicosom 58.

Granone F, (1967). L'ipnositerapia nel divezzamento degli alcoolisti. Rass Ipnosi Med Psicosom 58.

Granone F, (1980). Considerazioni psiconeurofisiologiche sul dolore desunte dalla pratica ipnotica. Arch Psicol Neurol Psich 61.

Granone F, (1980). Ipnositerapia in fobica, alcool-farmacodipendente, con spiccate anomalie erotiche. $2^{\circ}$ e $3^{\circ}$ Seminario I.C.S.A.T., Cissipat, Padova.

Granone F, (1983). Trattato di ipnosi. Boringhieri, Torino.

Hartman E, (1973). Biologia del sogno. Boringhieri, Torino. p. 189-214.

IEuD-Istituto Europeo Dipendenze. Terapia con TMS (Stimolazione Magnetica Transcranica). https://istitutoeuropeodipendenze.it/stimolazione-magnetica-transacranica/

INS - Istituto di Neuroscienze. Stimolazione magnetica transcranica (rTMS, Deep TMS, TMS Theta-Burst). https://istitutodineuroscienze.it/index.php/terapie/terapie-di-neuromodulazione/stimolazione-magnetica-transcranica-rtms/

Jessen, riferito da S. Freud, L'interpretazione dei sogni, op.cit. 
Kalenda V, (2019). Cancro, il termomagnetismo promette cure personalizzate. https://www.galileonet.it/termomagnetismonuovo-approccio-contro-tumori/

Koella W, (1975). Fisiologia del sonno. Il pensiero scientifico Editore, Roma.

Kono M, (2021). Gioco d'azzardo: uno studio sull'efficacia della Stimolazione Magnetica Transcranica (TMS). https://istitutodineuroscienze.it/index.php/2021/02/23/gioco-dazzardo-unostudio-sullefficacia-della-stimolazione-magnetica-transcranica-tms/

Lefaucheur JP, Obadia NA, Antal A, (2014). Evidence-based guidelines on the therapeutic use of repetitive transcranial magnetic stimulation (rTMS). Clin Neurophysiol 125:2150-206.

Mastronardi V, (1974). L'ipnosi nel quadro della psico-terapia. Rass Ipnosi Med Psicosom 10:27.

Mastronardi V, (1977). Dissuefazione dell'etilista con l'ipnosi (dinamismi psichici e tecniche). In: Prospettive psicoterapiche nel trattamento degli alcoolisti. Il pensiero scientifico Editore, Roma.

Mastronardi V, (1980). Nuove ricerche in tema di terapia ipnotica in un gruppo di etilisti cronici mediante immagini subliminali, I e II parte. Rasse Studi Psich 69.

Mastronardi V, (1982). Devianza e psicopatologia sociale (10 anni di ricerche in tema di ipnosi e training autogeno). Edizioni Levante, Bari.

Mastronardi V, Megna G, Cerfeda F, Bandiera L, Federici A, (1977). La percezione degli angoli articolari in neurolesi motulesi. Acta Neurol 32:323-31.

Mastronardi V, Puca FM, Passarella B, Vitiolo M, Minervini MG,
(1977). Influenzamento del racconto dei sogni da parte di immagini visive evocate verbalmente in sonno REM. Folia Neuropsichiatrica. Ed. Salentina, fascicolo 1-11.

Meier, riferito da Freud, "L'interpretazione dei sogni”, op. cit.

Overton DA, (1972). State dependent learning produced by alcohol and its relevance to alcoholism. Physiol Behav 2:197217.

Pallanti S, (2020). Stimolazione Magnetica Transcranica ripetitiva (rTMS). https://www.stefanopallanti.com/servizi/cosa-sonole-terapie-di-neuromodulazione/rtms/

Pavlov IP, (1966). I riflessi condizionati. Trad. M. Silvestri Lapenna, Boringhieri, Torino.

Puca FM, Passarella B, Mastronardi V, Vitolo M, Minervini MG, (1979). Esperienza di behaviour therapy durante il sonno REM in alcoolisti cronici. Riv Ital Elettroencefal Neurofisiol Clin 2.

Redazione Aboutpharma Online, (2019). Tumori, il termomagnetismo come supporto alle cure oncologiche. https://www. aboutpharma.com/blog/2019/06/20/tumori-il-termomagnetismo-come-supporto-alle-cure-oncologiche/

Schonbar R, (1965). Differential dream recall frequency as a component of lifestyle. J Cons Psychol 29:468-74.

Shevrin H, Luborski L, (1958). The measurement of preconscious perception in dreams and images: and investigation on the Poetzl phenomenon. J Abnorm Soc Psycol 56:468-474.

Sobell MB, Sobell LC, (1973). Alcoholics treated by individualized behaviour therapy: one year treatment outcome. Behav Res Ther 4:599.

Whitman R, (1963). Remembering and forgetting dreams in psychoanalysis. J Am Psychoanal 11:752-74.
Correspondence: Vincenzo M. Mastronardi.

E-mail: vincenzo.mastronardi@gmail.com

Key words: TMS; electromagnetic stimulation; therapy.

Parole chiave: TMS; stimolazione elettromagnetica; terapia.

Palabras clave: TMS; estimulación electromagnética; terapia.

Received for publication: 5 October 2020.

Accepted for publication: 28 October 2020.

This article is distributed under the terms of the Creative Commons Attribution Noncommercial License (by-nc 4.0) which permits any noncommercial use, distribution, and reproduction in any medium, provided the original author(s) and source are credited.

${ }^{\circ}$ Copyright: the Author(s), 2020

Licensee PAGEPress, Italy

Rivista di Psicopatologia Forense, Medicina Legale, Criminologia $2020 ; 25: 555$

doi:10.4081/psyco.2020.555 